region attacked by colicin E3, do not interfere with colicin inactivation.

Confirmation that the attack on the 16S RNA is indeed the lethal event, rather than any action in the membrane, for example, that might precede it, comes from Samson, Senior and Holland $(J$. Supramol Struct., 1, 135; 1972), who find that protein synthesis is arrested some five minutes after the addition of colicin E3 to the culture, and that the cleavage of the RNA, as followed by gel electrophoresis, occurs over about the same period. There is in fact some indication that protein synthesis continues for a few minutes after cleavage, perhaps until completion of the polypeptide chain. A curious observation is that a small amount of the $3^{\prime}$ terminal fragment, or at least something indistinguishable from it electrophoretically, appears to be present even in cells that have not been exposed to the colicin.

The other colicins, E1, E2 and $\mathrm{K}$, which are also proteins of about 60,000 molecular weight, do not evidently attack the ribosomes, and may act instead on the membrane. Like E3, they must have the ability to enter the cell, in spite of their large size. Direct observation of some form of mayhem by colicin $\mathrm{E} 1$ in the $E$. coli membrane is described by Phillips and Cramer (Biochemistry, 12, 1170; 1973). The fluorescence of the lipid-soluble molecule, anilinonaphthalene-sulphonic acid (ANS) in the $E$. coli membrane is slightly shifted and considerably enhanced on addition of colicin E3, and the same is true of the less polar, and presumably even more lipophilic species N-phenylnaphthylamine (NPN). The cells can be rescued from the effect of colicins by addition of trypsin within a short period after exposure to the colicin, which is presumably destroyed on the surface before its ingress into the cell. Phillips and Cramer find that the fluorescence enhancement lags behind the absorption of the colicin measured in this way. The colicin EI induces leakage of potassium and a drop in intercellular ATP concentration. This, however, is not the cause of the fluorescence change, for the perturbation is not affected by prior exposure to a reagent that causes the ATP level to be sustained. The simplest interpretation of these events is that the colicin brings about a cataclysmic change in the membrane when it enters, which affects permeability to potassium ions or ATPase activity.

In an accompanying article, Cramer, Phillips and Keenan (ibid., 1177) adduce more evidence that the fluorescent enhancement of the NPN is a structural effect, for the Arrhenius plot for the kinetics of the increase show a break that corresponds to the temperature of the membrane phase transition to a more fluid state, this depending on the temperature of cell growth, which in turn governs the phospholipid composition of the membrane. There seems also to be a small change in fluorescence polarization, and hence mobility of the fluorescent marker. The passage of the colicin into the membrane thus seemingly depends on the nature of the bilayer.

The colicins have specific membrane receptors, which evidently participate in their passage through the bilayer, and Sabet and Schnaitman (J. Biol. Chem., 248, 1797 ; 1973) have now achieved substantial purification of such a protein. The colicin E3 binding activity passes into a detergent-soluble fraction on disruption of the membranes, and can be further purified by ion-exchange chromatography. Not only does it bind the colicin, but the complex is precipitated by a colicin-directed antiserum. Moreover, the protein is missing in colicin E3-resistant cells. Sabet and Schnaitman estimate that there are some 220 molecules of the receptor per cell. The similarity between the colicins is strikingly underlined by the observation that the colicin E2 binds to the same receptor. Colicins E1 and $\mathrm{K}$, however, do not bind. The receptor activity for these species is also solubilized by the detergent, but does not remain with the E3 and E2-binding activity after fractionation. There are reasons for postulating that E1-receptor activity might involve the same protein (or actually glycoprotein, for carbohydrate residues are implicated) in combination with some other component.

\section{PLANT GEOGRAPHY \\ Success and Fall}

from our Plant Ecology Correspondent Much effort has been expended on tracing the geographical origins of domesticated plants and animals. Many species have been transported far from the places in which they evolved and their responses to climatic factors have been greatly modified by selective breeding. This makes it difficult to determine their origins from their physiological attributes. Weed species have certain features in common with crop plants; they too have been carried far from their points of origin, often to regions of differing climates. Weeds, however, have not been subjected by man to such rigorous selective pressures

\title{
Where the Rhodopsin is Not
}

A RECENT article by Dratz et al. (Nature New Biology, 237, 99 ; 1972), purporting to show that rhodopsin is completely buried within the rod outer segment membrane has drawn criticisms from Vanderkooi, and a rejoinder from Dratz and Schwartz. In the original article the inaccessibility of the rhodopsin at the aqueous interface of the membrane was inferred by the failure of reactive side chains to react with a covalent labelling compound incapable of penetrating into the bilayer. By contrast, rhodopsin liberated by disruption of the membrane reactcd copiously. Vanderkooi points out, in next Wednesday's Nature New Biology (April 18), that there could be other reasons for low reactivity than physical occlusion. He notes that other workers have found reagents capable of reacting with rhodopsin side chains in the membrane, supposedly without entering the bilayer. He also draws attention to a large body of X-ray diffraction evidence which is united in the one respect that the rhodopsin is only partly immersed in the membrane, and projects out of it into the aqueous medium.

In an accompanying communication, Dratz and Schwartz reject Vanderkooi's arguments, but not apparently his conclusion that their inferences were wrong. They suggest that published results on specific labelling referred to by Vanderkooi are also ambiguous, because some reagents can penetrate the bilayer and others have a geometry that may enable the reactive group to project into the bilayer and there react. They then refer to work of their own, and others, given at a recent meeting of the Biophysical Society, which indicates that part of the rhodopsin is in fact available to reagents in the aqueous medium. They have also calculated electron density profiles across the membrane for various dispositions of the rhodopsin, and do not seem to find good agreement with experiment for any of the models, including that for totally buried protein: here the shape is right (which it usually is), but the quantitative fit is poor.

The dispute may help to clarify the limitations in the use of labelling reagent for membrane proteins, includes an implicit admission of error in the earlier work, but makes no impression on the problem of rod outer segment membrane structure.

An article by Phillips and Morrison in the same issue of Nature New Biology emphasizes the complexity of reactivity of membrane components. Iodination of red cell membranes under the action of lactoperoxidase leads to labelling of only two proteins, which are therefore accessible at the surface. The new results show that after mild treatment with trypsin, which releases surface glycopeptides, the same two proteins take up much more label. This is interpreted in terms of a protective carbohydrate barrier on the outer membrane surface. 